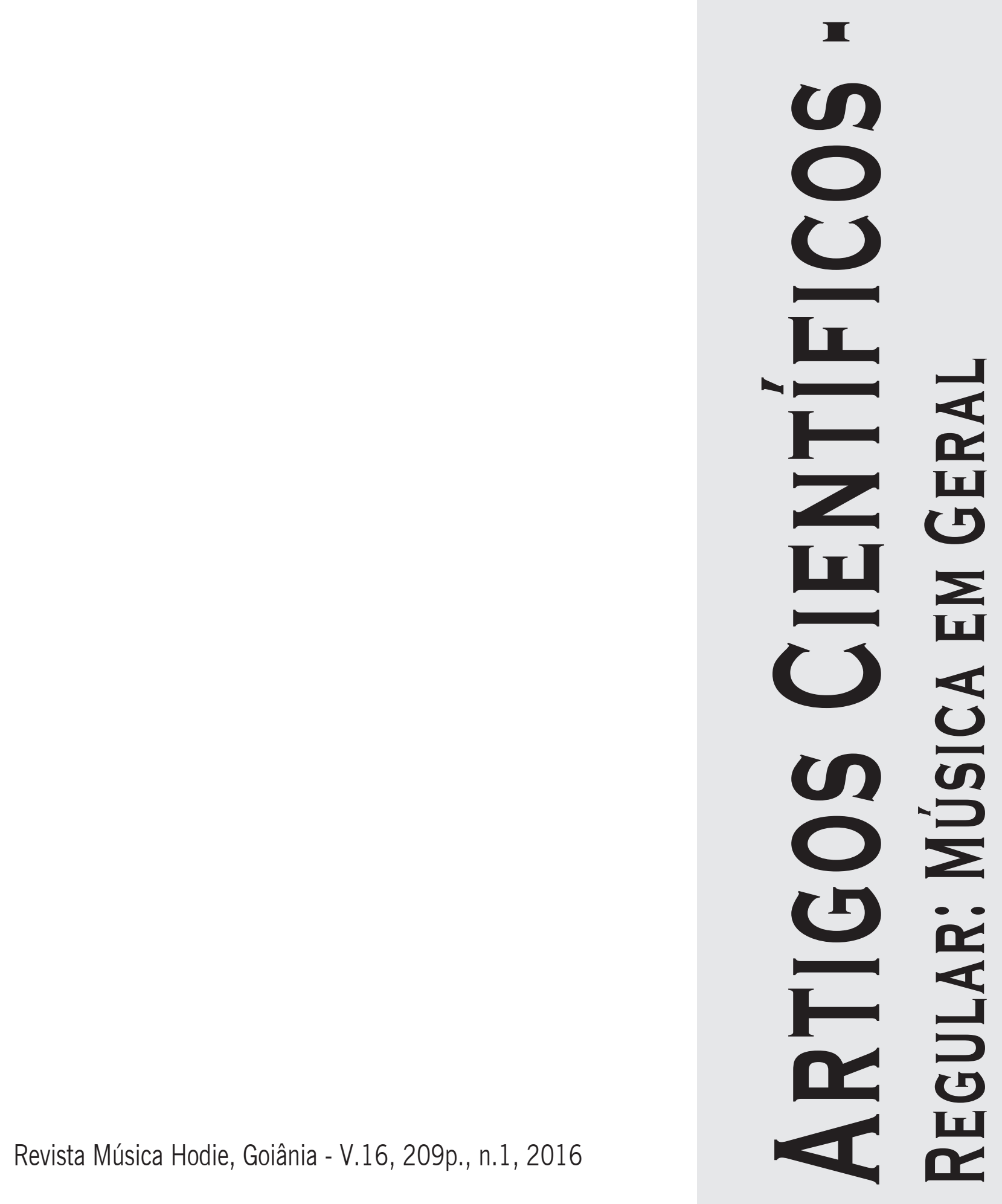




\title{
Resposta de orientação musical: uma hipótese para o dispositivo de origem do sentido
}

\author{
Marcos Nogueira (EM/Universidade Federal do Rio de Janeiro, Rio de Janeiro, RJ, Brasil) \\ mvinicionogueira@gmail.com
}

\begin{abstract}
Resumo: A neurociência cognitiva já nos oferece significativos indícios de que a função básica do cérebro é prever o futuro. Fazemos isto projetando coerente e simetricamente sentidos constituídos nas memórias de trabalho e de longo-prazo, a partir do que nos preparamos para novas experiências. Pretendo argumentar que a música é uma radicalização da experiência de previsão, sem o que não há sentido nem música. E a complexidade do ato da escuta musical implica uma multiplicidade de modos de abstração que regularão o que será percebido pelo ouvinte como relevante. O artigo propõe a hipótese de que o dispositivo cognitivo que dispara o processo de entendimento da música é o que poderíamos chamar resposta de orientação musical, associado ao conceito de novidade.
\end{abstract}

Palavras-chave: Cognição musical; Sentido musical; Resposta de orientação; Novidade em música.

Musical orienting response: A hypothesis for origin device of meaning

Abstract: Cognitive neuroscience already offers us significant evidence that the basic function of the brain is to predict the future. We do that by projecting coherent and symmetrically meanings constituted in working and long-term memory, from what we prepare for new experiences. I argue music is a radicalization of prediction experience, without which there is no meaning or music. The complexity of the act of musical listening implies a variety of abstraction ways that regulate what will be perceived by the listener as relevant. The article proposes the hypothesis of a cognitive device that triggers the music understanding process we might call musical orienting response, associated with the concept of novelty.

Keywords: Music cognition; Musical meaning; Orienting response; Novelty in music.

Respuesta de orientación musical: Una hipótesis para el dispositivo de origen del sentido

Resumen: La neurociencia cognitiva ya nos ofrece evidencia significativa de que la función básica del cerebro es predecir el futuro. Hacemos esto mediante el uso coherente y simétrico de la memoria a largo plazo y de trabajo, desde y hacia que nos preparamos para nuevas experiencias. Me gustaría argumentar que la música es una radicalización de la experiencia de la previsión, sin la cual no hay un sentido o la música. Y la complejidad del acto de escucha musical implica una variedad de modos de abstracción que rigen lo que va a ser percibido por el oyente como relevante. El artículo propone la hipótesis de que el dispositivo cognitivo que desencadena el proceso de la comprensión de la música es lo que podríamos llamar la respuesta de orientación musical, asociado con el concepto de novedad.

Palabras clave: La cognición musical; Significado musical; Respuesta de orientación; Novedad en música.

\section{Apresentação}

A "resposta de orientação" (orienting response), também conhecida como "reação de orientação" (orienting reaction) ou "reflexo de orientação" (orienting reflex), é uma ação imediata do organismo em resposta a uma mudança particular que percebe em seu meio ambiente. Uma característica marcante da resposta de orientação é que ao perceber o evento que extrapola dado limiar de discrepância no meio circundante o indivíduo dirige sua atenção ao evento antes mesmo de identificá-lo. Podemos entender a resposta de orientação como um conjunto de indicadores corporais responsivos que sinalizam a percepção de um estímulo que se sobressai como relevante. $\mathrm{O}$ fenômeno seria, entretanto, uma resposta à novidade "não aversiva". Por exemplo, no domínio auditivo um evento sonoro percebido como novidade provocará resposta de orientação espontânea, a menos que se apresente em nível de intensidade sonora tão extraordinário que supere a zona de conforto auditivo e inflija ao indivíduo uma espécie de ameaça. E esta última situação provoca outro tipo de reação entendida genericamente como "reflexo defensivo" (defensive reflex) do indivíduo, que emerge 
como ação de "bloqueio" ao evento ameaçador. Enfim, simples respostas de orientação ocorrem quando a mudança percebida no meio circundante não provoca outro tipo de reação conhecida também como "reação de sobressalto" (startle reaction) ou "reflexo de sobressalto" (startle reflex), o susto.

O fenômeno da resposta de orientação foi primeiramente descrito pelo fisiologista russo Ivan Sechenov, em seu Reflexes of the brain (1863/1965), mas foi Ivan Pavlov que em Conditioned reflexes: An investigation of the physiological activity of the cerebral cortex (1927) o identificou nos termos em que é até hoje estudado - Pavlov também referiu o fenômeno como "reflexo de 'o que é isto?" -, observando que a percepção de novidade ou mesmo de um evento significativo é a causa central do fenômeno. Para ele, diante da novidade o indivíduo interrompe imediatamente o que está fazendo e dirige seus recursos cognitivos para a fonte da estimulação - trata-se, portanto, de um componente comportamental de orientação.

O presente estudo aborda o processo de formação do sentido musical. A literatura em teoria da música que dialoga com os resultados da psicologia experimental, sobretudo desde Emotion and meaning in music (1956) de Leonard Meyer até Sweet anticipation (2006) de David Huron, vem ressaltando o papel da emoção e da expectativa (sobretudo a capacidade de anteciparmos imaginativamente a ocorrência de certos eventos) e suas implicações como experiências primordiais que condicionam a emergência do sentido musical. Pretendo propor a mudança deste foco na investigação do sentido produzido no ato da escuta musical, recuperando o conceito "comportamentalista" de resposta de orientação, aqui, porém, abordado no contexto da semântica cognitiva de base enacionista. A hipótese primeira do trabalho é que, ao longo da experiência da música, não a apreendemos "por expectativas", não nos mantemos motivados ao engajamento com a música regulados, simplesmente, por crenças e antecipações imaginativas de eventos futuros da obra que experimentamos - atos mentais mais e menos conscientes e, em grande parte, condicionados pela expertise do ouvinte, impulsionados pelo desejo da recompensa de sentido. Ao invés, quero crer que no desenrolar da experiência da escuta os condicionamentos estilísticos e contextuais - esquemas ${ }^{1}$ cognitivos de memória - com os quais enfrentamos uma nova experiência musical não devem ser confundidos com expectativas e antecipações. Argumento que, ao longo da escuta musical, as experiências de expectativa são de fato pontuais. Ao invés, são os eventos percebidos como "novidade", aqueles que no ato da escuta são avaliados como "inesperados", que normalmente revelam ao ouvinte que este nutria a expectativa de outro evento em seu lugar.

Eventos musicais inesperados provocam respostas de orientação do ouvinte. Se provocam respostas de orientação é porque são inesperados, e se são inesperados é porque se opõem a expectativas de outros eventos. Acredito que o efeito de negação à continuação esperada é tanto mais intenso quanto o grau de discrepância, de inesperabilidade do evento. Com frequência só dirigimos atenção especial à novidade e nos tornamos conscientes de que possuíamos a expectativa de outra condição de continuidade, quando o grau de discrepância do evento é significativo. De outro modo a resposta de orientação ao evento inesperado apenas dá início a uma simples atualização do modelo conceitual adotado para orientar a produção de sentido e não experimentamos, propriamente, o efeito da expectativa. O presente estudo discute que o processo de construção do sentido musical é implacavelmente determinado pelas contingências da interação entre um complexo de eventos potencialmente relevantes da obra musical - as affordances de Gibson (1979) - e um ouvinte executante de ações que podemos entender como respostas de orientação musicais, em consequência das quais enfocaremos ou não nossas expectativas e produziremos antecipações. 


\section{Novidade e processo atencional}

Novidade, enquanto coisa que representa alguma mudança (descontinuidade) significativa no meio ambiente - ou, particularmente, no fluxo musical -, nem sempre é algo de fácil identificação ou classificação, bem como a significância de um evento é uma questão que também impõe alguma dificuldade para uma teoria da resposta de orientação, pois a significância de um estímulo pode ser "inata" (considerando os reflexos defensivos, por exemplo, como descritos pelas teorias evolucionistas, que explicam que a resposta condiciona o organismo à execução de futuras ocorrências semelhantes) ou "aprendida" (como o timbre da voz de alguém ou o som do nome de alguém, sobretudo do próprio indivíduo "estimulado"). Embora seja importante para os organismos detectarem novidades em seus meios, não há como prever antecipadamente quando um evento será relevante como modificador das condições do meio. No caso particular da escuta musical, é frequente depararmos com situações em que atribuímos, inicialmente, relevância a certos eventos de uma obra que ouvimos pela primeira vez e que, ao longo da experiência, mostram-se irrelevantes. As pesquisas vêm possibilitando considerar a hipótese de que quanto maior a densidade de eventos "novos" em dado trecho do fluxo musical, maior a possibilidade de algum desses se tornar significativo no ato da escuta. Desse modo, trechos musicais com poucos eventos potencialmente "novos" seriam potencialmente menos difíceis de assimilação, uma vez que se apresentam como configurações de caráter fluido e consistentemente coerente, suscitando menos esforço de atenção do ouvinte em seu contínuo processo de entendimento. Enfim, se o fluxo musical não proporcionar desafios de reorientação ao ouvinte, deste exigirá menos atenção - que aqui entenderei como menos interesse. Por outro lado, se tudo no fluxo musical parecer novidade ao ouvinte, a música se apresentará como insuperavelmente confusa.

Pavlov havia descoberto que depois de repetidas apresentações de um "novo" evento, este não mais provocava o "reflexo condicionado". Anos depois, Evgeny Sokolov (1960, 1963) dedicou-se sistematicamente ao fenômeno, descrevendo ainda o principal objeto de suas investigações: a habituação enquanto processo de familiarização gradual com um evento novo que se torne repetitivo. Segundo ele, a repetição do evento proporciona uma redução progressiva da ativação de respostas de orientação. Assim, a introdução original de uma mudança no modelo neuronal atualmente ativo (currently active neuronal model), ou seja, aquele em que o indivíduo está focado, resulta em resposta de orientação; porém as ocorrências subsequentes do mesmo evento motivarão respostas de orientação cada vez menos intensas e, eventualmente, cessarão. Portanto, ao familiarizar-se com o evento o indivíduo passa, gradualmente, a avaliá-lo como inconsequente e desimportante, e passa a não mais alocar esforço de atenção ao mesmo. Sokolov fez também outra descoberta importante ao enfocar o que denominou desabituação. Ele apresentou a um indivíduo um som com determinada altura (nota) e registrou uma resposta de orientação. Como previra, com as consecutivas apresentações do mesmo evento a resposta de orientação gradualmente desapareceu. No entanto, ao apresentar um som com uma nova altura (mais aguda ou mais grave) ou com um novo padrão de intensidade sonora (mais forte ou brando) a resposta de orientação reapareceu. Esta nova condição resultaria, segundo ele, de desabituação.

Pavlov presumiu que a resposta de orientação seria um reflexo incondicionado à novidade, entendimento que se disseminou até Grastyan (1961) argumentar que seria mais adequado considerá-la uma reação condicionada. Em suas pesquisas Grastyan baseou-se em algumas considerações: (1) o fato de que estímulos não familiares ao indivíduo não evocam, propriamente, respostas de orientação, estas que só surgiriam após o estímulo já ter 
se tornado habituado e então desabituado, pois estímulos completamente inesperados provocariam, propriamente, a reação de sobressalto, uma súbita reação defensiva ao estímulo, desprovida do caráter investigativo do reconhecimento, próprio da verdadeira resposta de orientação; (2) a resposta de orientação pode ser facilmente habituada, assemelhando-se a reações condicionadas, ao invés de reações incondicionadas; (3) a ausência de resposta de orientação nas primeiras semanas após o nascimento do indivíduo e sua emergência algum tempo depois pode sugerir que é essencialmente aprendida, considerando ainda que antes de a resposta de orientação se apresentar como fato, o equipamento motor oferece suporte a reações defensivas.

Considerando a relação direta entre o evento inesperado e o processo atencional, é necessário salientar que atenção aqui pode ser entendida com a “alocação de recursos” mentais para tratar um estímulo específico. Considerando a limitação desses recursos, quanto mais atenção um evento requerer menos disponibilidade o indivíduo terá para realizar outras tarefas. Enfim, isto impõe ao indivíduo um contínuo processo de realocação de reservas cognitivas no processo de abstração e entendimento. O termo “atenção” é aplicável a diversos objetos. Para Shiffrin (1988), "refere-se a todos os aspectos da cognição humana que o sujeito pode controlar e a todos os aspectos da cognição relacionados com recursos ou capacidade limitados e métodos de lidar com tais restrições” (p. 739) ${ }^{2}$. Portanto, estamos tratando de uma capacidade limitada para processar os dados da consciência e que esta alocação pode ser intencionalmente controlada.

Desde a fase inicial da psicologia experimental a atenção tem sido considerada um processo de assunção consciente do controle da mente. Entretanto, os estudos desenvolvidos nas últimas décadas enfocam o dispositivo da atenção em diferentes paradigmas. A atenção "definida por tarefa" (task-defined) é um método puramente descritivo da atenção, demonstrada quando o sujeito é capaz de cumprir satisfatoriamente uma tarefa que dele exigiu a seleção de um estímulo específico em detrimento de outros estímulos presentes. Ou seja, a atenção é inferida com base na qualidade do cumprimento de uma tarefa que teria exigido do sujeito o isolamento de dado estímulo. Contudo um entendimento da atenção como processo psicológico, propriamente, descreve-a como atividade de focalização da mente, "orientada ao processo" (process-oriented), um dispositivo ativo de seleção de um dentre muitos possíveis estímulos sensoriais ou encadeamentos de pensamento, com o fim de otimizar a qualidade do dado enfocado e a efetividade do processo mental. Assim a atenção seria necessária quando diante de uma extraordinária densidade de estímulos e tarefas os processos mentais exigem-na para operar de modo satisfatório.

A maior parte das pesquisas em torno da atenção orientada ao processo vêm enfocando a atenção espacial. Inúmeras pesquisas desenvolvidas nas últimas décadas dos novecentos em torno da experiência visual demonstraram que a explícita reação de sobressalto a eventos súbitos, intensos e inesperados é espelhada por uma orientação da atenção que pode ser disparada automaticamente por um súbito, embora não necessariamente "intenso", transiente sensorial (a mera novidade). Devemos aqui atentar que seja qual for o evento discrepante que provoque respostas de orientação automáticas do indivíduo, não representa, ele próprio, uma ameaça ou acontecimento relevante, mas está, provisoriamente, no lugar de possíveis ameaças ou simples acontecimentos subsequentes. Isto é, algo importante a se considerar é que a atenção é capturada sempre que o sistema detecta a presença de novidade e que o que há de motivador nos estímulos que capturam a atenção é, em tese, o fenômeno da descontinuidade (JONIDES, YANTIS, 1988; HILLSTROM, YANTIS, 1994; YANTIS, EGETH, 1999; FOLK \& REMINGTON, 1999). Há indícios significativos de que este fenômeno reflete uma orientação "intencional" da atenção e isso abre caminho para a hipótese de que 
um entendimento completo dos fatores que determinam se dado evento automaticamente capturará a atenção do indivíduo pode ser virtualmente inalcançável - considerando-se, por exemplo, fatores de habituação e condicionamentos culturais -, questão esta crucial em grande parte dessas pesquisas (ATCHLEY, KRAMER, HILLSTROM, 2000; FOLK, REMINGTON, 1999; GIBSON, KELSEY, 1998; YANTIS, EGETH, 1999).

Como já haviam observado Kahneman e Henik (1977), no contexto experiencial da visão estamos, em geral, mais preocupados com os objetos do que com as localizações que eles ocupam. Muitos estudos, desde então, vêm dedicando esforços no sentido de distinguir os processos atencionais baseados "em objeto" e baseados "em espaço" (DUNCAN, 1984; EGLY, DRIVER, RAFAL, 1994). Dado que os objetos se apresentam em localizações específicas, não é fácil discriminar a alocação da atenção ao objeto e à sua localização, dados densamente confundidos. Donde resulta um entendimento de que há uma multiplicidade de níveis de atenção incluindo tanto atenção por objeto quanto por espaço. A ênfase, ora de um ora de outro dado perceptivo, tem levado pesquisadores como Vecera (2000) a propor que a seleção de estímulos baseada em objeto está intimamente relacionada à segmentação do objeto. A segmentação da cena visual envolve a discriminação dos atributos que concorrem para formar um objeto em contraste com os demais objetos à sua volta, ou seja, a habilidade para distinguir "figuras" sobre um "fundo". Isso implica considerar que a atenção baseada em objetos seleciona uma forma segregada dentre diversas outras formas segregadas, ou mesmo enfoca uma forma específica contra a divisão da atenção a diversas formas concomitantes. Alguns estudos têm abordado relações entre dispositivos de seleção baseada em objeto e em espaço (KRAMER, JACOBSEN, 1991; VECERA, 1994; VECERA, FARAH, 1994). Os resultados relatam que a localização espacial dos objetos influencia a seleção, ou seja, em algumas tarefas é mais fácil dividir a atenção entre os objetos, quando estes são espacialmente próximos. Isso implica, portanto, que o processo de atenção baseada em objeto produz seleções a partir de segmentações perceptivas de uma cena espacialmente organizada pela mente.

A atenção seleciona subitamente estímulos, objetos e localizações espaciais ocorrentes em diferentes modalidades sensoriais. A questão que emerge, contudo, refere o controle atencional: como estes eventos se tornam relevantes para a seleção? Um evento abrupto de descontinuidade que provoque a alocação de recursos de atenção também determina a alocação de atenção para estímulos subsequentes? O controle atencional é relativamente óbvio quando apenas um evento se apresenta como potencialmente discrepante. Porém esta situação se torna menos controlável e previsível diante de uma cena complexa, um meio com múltiplos objetos de relevância compatíveis, que competirão pela atenção do indivíduo, demandando algum tipo de controle para resolver o problema de segmentação e seleção.

Desimone e Duncan (1995) propuseram uma estrutura para a conceituação do controle atencional. Trata-se do modelo de competição influenciada (biased competition model) no qual duas classes de parâmetros influenciam o controle atencional: os parâmetros "de baixo para cima" (bottom-up), baseados em estímulo, e os "de cima para baixo" (top-down), dirigidos a alvo, revelando-se assim estratégias distintas de fluxo no processo perceptivo (YANTIS, 2000). Segundo o modelo, parâmetros bottom-up incluem a súbita aparição de um estímulo - que quero aqui entender como um transiente sensorial - ou descontinuidade no meio; parâmetros top-down incluem tanto um padrão alvo, ou seja, uma representação mental do alvo que está sendo visado (DESIMONE, DUNCAN, 1995; DOWNING, 2000; DUNCAN, HUMPHREYS, 1989) - que ressalvo aqui poder ser tanto uma representação conceitual, propriamente, quanto um esquema cognitivo ativado inconscientemente no ato percepti- 
vo -, quanto as intenções do indivíduo acerca da configuração espaço-temporal do estímulo resultante de seus condicionamentos. Em uma rede de seleções excludentes, representações mais fortes tenderão a suprimir outras representações. Assim sendo, qualquer fator bottom-up ou top-down que reforçar dada representação dará a esta uma vantagem competitiva no sistema de representações neurais. De acordo ainda com o modelo, o cérebro gera um padrão atencional, um modelo conceitual, que representa demandas e objetivos da tarefa em andamento. Desse modo, quando o indivíduo realiza uma tarefa de busca de dado alvo um padrão atencional especificará a forma e a localização provável do alvo. As representações sensoriais envolvidas são comparadas ao padrão e aquelas que coincidem com o padrão são reforçadas. Além disso, fatores bottom-up tais como "contraste" também influenciam o reforço de representações. Enfim, tanto fontes bottom-up quanto top-down, em conjunto, influenciam a competição entre estímulos.

Luck e Vecera (2002) argumentam que os processos atencionais são úteis sobretudo quando dado sistema cognitivo enfrenta um afluxo de estímulos concorrentes, beneficiando-se da redução desses estímulos pela atenção. Esse tipo de sobrecarga de estimulação ocorre devido à limitação dos processos cognitivos, que operam com eficiência reduzida quando a quantidade de estímulos concomitantes potencialmente relevantes aumenta. A seleção operada pelo processo atencional reduz estrategicamente a quantidade de decisões que precisam ser tomadas pelo indivíduo, favorecendo sua acurácia. É provável que o termo atenção se aplique a muitos processos distintos que operam em subsistemas cognitivos diferentes, regulados pelas estruturas cognitivas e demandas próprias de cada subsistema. É essencial, todavia, distinguir o processo que dispara o direcionamento da atenção - a resposta de orientação - do processo que mantém a atenção, uma vez que há evidências de que podem ser distintos.

O presente trabalho enfoca estritamente os mecanismos que disparam a alocação de atenção no ato da escuta musical - a manutenção da atenção, que não é aqui discutida, é outro processo que exige abordagem própria e que está mais relacionado às experiências de expectativa e antecipação. Diante do exposto cumpre investigar a natureza dos fatores bottom-up e top-down que disparam o que quero reconhecer como respostas de orientação musicais e a alocação de recursos atencionais implicada no ato da escuta.

\section{O processo de organização perceptiva}

Assumindo os pressupostos da ciência cognitiva "ecológica”, pretendo relacionar os fatores bottom-up e top-down acima referidos aos reguladores da experiência interativa de produção de sentido musical. Quero argumentar que fatores bottom-up envolvidos nos atos de entendimento musical podem ser relacionados a affordances ${ }^{3}$, que restringem a ação imaginativa do indivíduo ao universo de sinalizações dispostas pelo fluxo musical - nas circunstâncias, é claro, em que a música é experimentada -, enquanto fatores top-down podem ser associados a esquemas cognitivos de memória, estruturas fisiológicas especializadas e condicionamento cultural do indivíduo. Se pretendo abordar as respostas de orientação em música como origem do processo semântico musical, a questão da natureza dos fatores que regulam o processo atencional no ato da escuta constitui foco central de investigação. E se estamos referindo dados perceptivos, cumpre discutir o conceito de organização perceptiva, que pode ser entendida tanto como estrutura experiencial baseada em atividade sensorial quanto como processo cognitivo subjacente que produz aquela estrutura. 
O problema central enfrentado pelo sistema nervoso visual (PALMER, 2002) como para o sistema nervoso auditivo (BREGMAN, 1990) é determinar uma estrutura para a imagem, ou seja, enfocar os componentes da cena visual ou auditiva que formariam os eventos, no sentido de corresponderem a um mesmo evento, a parte de um evento ou a um fluxo de eventos no meio. Assim sendo, a essência da organização perceptiva pode ser entendida como o processo pelo qual constrói-se uma hierarquia parte-todo para uma imagem ou para uma cena completa. É necessário, portanto, entender que tipo de estrutura parte-todo os indivíduos percebem em dada cena e como deveria ser caracterizada. Há, evidentemente, inúmeras possibilidades de organização para cada experiência perceptiva, mas a questão aqui é discutir como tal hierarquia parte-todo é estabelecida pelo sistema cognitivo. Para tanto cumpre entender a natureza dos estímulos selecionados pelo sistema para organizar a cena percebida. Isso inclui não apenas o exame da variabilidade dos fatores envolvidos nos atos perceptivos como também identificar os parâmetros que estabelecem potencialmente a relevância dos estímulos na constituição da estrutura perceptiva.

A tradição empirista da Modernidade, fundada, sobretudo, nas pesquisas de Locke e Hume, abordou a organização perceptiva como um processo aprendido por meio da regularidade de certos estímulos. Essa teoria estruturalista da percepção entendeu que o ato perceptivo surge de um processo no qual elementos sensoriais básicos evocam outros dados sensoriais já memorizados por repetição de ocorrências prévias. Assim, a relação entre sensações "simples" e a percepção de entidades complexas era entendida como análoga à relação entre átomos e moléculas (PALMER, 2002). Portanto, os estruturalistas defendiam a tese de que aquilo que mantinha as sensações juntas em perceptos mais complexos eram as associações aprendidas, que resultavam da contiguidade espacial e temporal daquelas sensações em experiências passadas.

A teoria da organização perceptiva foi então atualizada pelo notável empreendimento da psicologia da Gestalt (KOFFKA, 1935), que se opôs à visão tradicional de percepção como simples concatenação de elementos sensoriais, alegando que o exame desta concatenação não revelaria o sentido do todo perceptivo. Para os gestaltistas os perceptos complexos têm suas estruturas intrínsecas que não podem ser reduzidas a partes ou relações entre partes - a doutrina do holismo. Neste contexto a evidência holística é revelada pelas propriedades emergentes das configurações, inexistentes em qualquer das partes componentes do percepto. E o princípio central da teoria da Gestalt para a organização perceptiva foi o princípio da pregnância (Prägnanz), segundo o qual um percepto (uma segmentação consistente que o sistema nervoso faz do meio) será tão bom (relevante, dada a sua simplicidade e regularidade apreendidas) quanto as condições prevalecentes (a regulação imposta pelas limitações do sistema sensorial) possibilitarem. Os principais representantes dessa corrente tentaram fundamentar essas tendências a partir da estruturação do cérebro e de sua interação com a estimulação do meio (KÖHLER, 1947/1980). Assim sendo, no âmbito dessa teoria da forma o conceito de organização perceptiva está fortemente associado à ideia de segmentação do mundo, de agrupação formando unidades perceptivas mais relevantes do que outras possíveis. A agrupação não seria a única estratégia de organização perceptiva, mas provavelmente a mais importante, e Max Wertheimer, um dos fundadores da psicologia da Gestalt publicou, em 1924, artigo acerca desses mecanismos. Ele apresentou-os na forma de leis de agrupação: proximidade, similaridade, movimento comum, simetria, paralelismo, continuidade, fechamento, além de desdobramentos e combinações desses fatores.

O sentido de "simplicidade" e as estratégias que o cérebro usaria para selecionar o percepto mais simples não foram, todavia, suficientemente esclarecidos pelos psicólogos 
da Gestalt. O paradigma ecológico da psicologia cognitiva, que emergiu definitivamente, nos anos 1970, a partir das pesquisas de James J. Gibson (1977, 1979), Eleanor Rosch e colegas (1976, 1978), e outros, tentou assim superar as lacunas deixadas pelas teorias da Gestalt. A tese ecológica para explicar fenômenos perceptivos organizacionais tais como a seleção, a agrupação, a segmentação ou a segregação dos estímulos do meio considera certos recursos cognitivos de estruturação da cena em objetos (ou eventos) e partes. O princípio da homogeneidade e do padrão, como a similaridade de cor dos objetos físicos ou, por projeção metafórica, a similaridade de altura ou timbre dos objetos musicais - como discutiremos adiante -, é um fator relevante de agrupação, tendo em vista que os objetos e as suas partes tendem a ser razoavelmente homogêneos enquanto unidade. O mesmo princípio da homogeneidade pode ser aplicável à experiência de movimento no espaço, uma vez que os objetos enquanto agrupações apreensíveis tendem a se mover consistentemente como corpos unitários, de modo que estes movimentos projetados apresentam similaridade em direção e velocidade, favorecendo assim a organização perceptiva - o mesmo pode ser considerado no contexto fenomênico do espaço musical com respeito à percepção de movimento e direcionalidade.

Há uma questão crucial em psicologia cognitiva ecológica - fortemente presente nos estudos das ciências cognitivas incorporadas em geral - que, como Stephen Palmer (2002) atentou, já havia sido sinalizada no trabalho de Wertheimer: a experiência passada. Este dado lança a hipótese de que o ato de agrupação pode não ser a etapa inicial do processo perceptivo; a agrupação teria lugar a partir da interação de estímulo e conteúdos na memória - alguns preferem abordar a questão como "condicionamento", outros, como "cultura". A Gestalt e as subsequentes correntes cognitivistas representacionistas entenderam que esse conteúdo na memória são representações de experiências anteriores. A ciência cognitiva incorporada, por sua vez, corrente contemporânea essencialmente não representacionista, não considera que todo sentido seja representacional, sobretudo os sentidos pré-conceituais que constituem essencialmente esquemas cognitivos na memória. E nos termos desta corrente enacionista poderíamos reconstruir o princípio gestaltista da pregnância considerando que um percepto, enquanto segmentação consistente que emerge na interação de meio e sistema perceptivo, torna-se relevante em virtude tanto da simplicidade (homogeneidade) e regularidade (simetria de padrão) dos estímulos selecionados pelo sistema no ato perceptivo quanto das condições prevalecentes, na forma de uma ampla regulação determinada pelas disponibilidades restritivas (affordances) impostas pelo meio e pelas limitações dos recursos apreensórios do sistema perceptivo.

Os processos de segmentação da cena perceptiva, em geral, incluindo atos de seleção, agrupação e segregação - que podem ser entendidos tanto em sua similaridade quanto a partir de suas particularidades - seriam regulados por pregnância. Porém, que elementos conferem relevância a uma ou à outra configuração abstraída pelo indivíduo como região segregada do todo, em sua interação com um mundo pleno de disponibilidades concorrentes? Este particionamento da cena perceptiva em regiões fronteiriças segregadas e mutuamente excludentes vem sendo amplamente investigado, no campo da percepção visual, como fizeram Palmer e Rock (1994), que postularam que a segmentação de regiões é determinada por um princípio organizacional que denominaram conectividade uniforme. Eles entenderam que o primeiro passo na construção da hierarquia parte-todo de uma imagem visual é particioná-la em regiões conectadas uniformemente. E, para eles, ser "conectada uniformemente" é constituir um subconjunto do todo, simples e consistentemente uniforme ou, ao menos, que varie progressivamente em suas propriedades perceptivas como cor, textura, movimento, dentre outras. Enfim, nossa percepção da cena, globalmente, como dos 
objetos na cena, consistiria usualmente no particionamento da cena ou de seus objetos em várias regiões uniformemente conectadas.

Nesse sentido, quero entender que a superação de um limiar de discrepância de uniformidade que implique a quebra de conectividade entre regiões estabelece a segregação de objetos na cena. Para Palmer (2002), há duas maneiras básicas de tratar a tarefa de particionar uma imagem visual em regiões uniformemente conectadas, que constituem modos distintos de processamento neuronal: detectar diferenças em propriedades visuais e abstrair similaridades entre porções adjacentes da imagem. Assim considerando e investigando quais elementos da cena perceptiva poderiam emergir nesse processo interativo de percepção que regula nossas inferências de segmentação, diversos estudos reaproximaram-se notavelmente dos postulados da Gestalt, partindo da ideia de que formas "simples e regulares" seriam abstraídas da emergência de (1) contornos constituídos por continuidade e simetria de padrão, que estabelecem possibilidades de segregação por separação de regiões adjacentes (CANNY, 1986; MARR, HILDRETH, 1980), ou de (2) regiões (LEUNG, MALIK, 1998) constituídas por homogeneidade e regularidade, e que estabelecem possibilidades de segregação basicamente por contraste textural (BECK, 1982; JULESZ, 1981).

\section{Resposta de orientação e a origem do sentido musical}

É crucial para um organismo apreender o meio acústico em torno de si, constituído por um conjunto de efeitos de fontes sonoras - objetos que vibram devido a ações físicas sobre eles -, a fim de se capacitar a estruturar seu entendimento e comportamento em relação a esta cena. Abstraímos os vários efeitos sonoros concorrentes numa cena auditiva (BREGMAN, 1990) como eventos independentes um do outro, que podem, entretanto, interferir na percepção dos demais, caso seu efeito seja, por exemplo, muito mais intenso que os dos outros, determinando a densidade da cena em questão. A resposta de orientação ocorre quando o indivíduo busca o entendimento da experiência atual na memória de curto-prazo (memória de trabalho) e esta operação falha, induzindo o aparelho cognitivo à ativação de recursos da memória de longo-prazo, com eventual atualização consecutiva desses conteúdos na memória. Reisenzein, Meyer e Schützwohl (1996) entenderam o termo como uma síndrome probabilística de respostas provocadas, em particular, pela novidade, incluindo vários componentes comportamentais e fisiológicos. Abordando o que entenderam serem as condições sob as quais se dá a experiência da novidade, Reisenzein, Meyer e Niepel (2012) propuseram, a título de exemplo, que um evento musical simples, uma nota, apresentada "pela primeira vez" ao ouvinte, seria uma novidade num primeiro sentido; uma nota percebida como desvio ocasional, uma nota "estranha" que impede a ocorrência de dada sequência melódica esperada, seria uma novidade num segundo sentido; e um contorno melódico que não segue o padrão de continuidade estabelecido pelos contornos anteriores, contradizendo-os, seria uma novidade num terceiro sentido.

Diversas pesquisas vêm investigando os modos como o sistema perceptivo interage com o meio sonoro para estruturá-lo (BREGMAN, 1990, 1991; MCADAMS, DRAKE, 2002). No presente estudo entendo que a organização perceptiva dos eventos sonoros concorrentes numa cena auditiva pode resultar em: (1) fusão perceptiva, quando dois ou mais eventos potencialmente distintos são percebidos como um único evento; em (2) agrupação perceptiva, quando componentes da cena auditiva percebidos como eventos independentes são agrupados perceptivamente formando contornos e regiões distinguíveis; e em (3) segregação perceptiva, quando os componentes complexos da cena (agrupações) são percebidos 
como subfluxos concorrentes de um fluxo complexo. Evento auditivo refere aqui uma unidade sonora de extensão temporal limitada, experimentada quando fontes sonoras são colocadas em vibração por ações físicas - é necessário observar que uma mesma fonte pode produzir efeitos sonoros percebidos como simples ou complexos. Os resultados da pesquisa em torno da organização perceptiva da cena auditiva vêm reiterando que há um número relativamente limitado de tipos de pistas (cues) acústicas que sinalizam a constituição da cena em seus componentes. Essas pistas indicam diversos atributos da cena, regulando as possibilidades de sua apreensão pelo indivíduo. O entendimento daquilo que ocorre na cena também resultará da intenção perceptiva e dos recursos cognitivos e culturais que o perceptor envolve no processo. Desse modo, algumas pistas são mais efetivas na agrupação de componentes da cena, de acordo com algum atributo acústico do evento auditivo, propriamente, tal como seu conteúdo tonal (seja ele constituído por alturas precisamente identificáveis ou apenas pela identificação de registros aproximados), ou de acordo com atributos das fontes sonoras, tal como sua posição no espaço físico.

O foco do presente estudo são os atos de apreensão - respostas de orientação - dessas pistas acústicas na experiência da música, um processo que entendo ocorrer em concomitância com os processos de fusão de evento e de agrupação de eventos, determinantes para a segregação do fluxo musical. Trata-se da investigação de como respostas de orientação musicais sinalizam a presença de tais pistas (sejam estas um evento simples, uma agrupação pontual ou o ponto de início de uma agrupação sequencial mais extensa), a partir do que terão início, propriamente, os processos de segregação da cena musical. É necessário, neste ponto, investigar que tipos de pistas acústicas provocam respostas de orientação na experiência da música. Diversos estudos vêm dedicando atenção ao processo de organização perceptiva da cena auditiva musical, desde os anos 1980, sobretudo voltados para os campos "harmônico" e "temporal" (MOORE, GLASBERG, PETERS, 1985; PALMER, KRUMHANSL, 1987; HARTMANN, 1988; BREGMAN, 1990; HARTMANN, MCADAMS, SMITH, 1990; ROBERTS, BREGMAN, 1991; ZERA, GREEN, 1993; MCADAMS, BOTTE, DRAKE, 1998). Proponho considerar aqui uma classificação básica de atributos constituintes das pistas acústicas musicais em: (1) tonais, considerando os conteúdos sonoros relativos à percepção de alturas determinadas (ou relativamente determinadas) e às condições de compatibilidade entre os conteúdos tonais dos componentes da cena musical por continuidade, homogeneidade, regularidade e simetria; (2) texturais, considerando tanto as envoltórias de intensidade sonora e o comportamento espectral (timbre) dos eventos da cena quanto à densidade do fluxo (com respeito à complexidade de segregação), porque percebemos mudanças conjuntas e comuns desses parâmetros como constituindo pistas para fusão e agrupação sequencial dos componentes da cena musical, ao passo que mudanças independentes e dissimilares (não "paralelas”) tendem a sinalizar potenciais segregações do fluxo; (3) temporais, tendo em vista que eventos sonoros raramente têm início ou desaparecem exatamente no mesmo instante e, por isso, o sistema perceptivo presume que componentes da cena percebidos como absolutamente sincrônicos devem ser fundidos como elementos de um mesmo evento, enquanto componentes assincrônicos tendem a gerar "movimento" e ser agrupados sequencialmente; e (4) topográficos, considerando a ênfase dada à espacialidade dos componentes da cena, um mapeamento de posições no espaço fenomênico do fluxo musical, que emerge no ato da escuta quando o processo atencional sobrepõe a posição espacial do evento musical ao próprio evento.

O sistema auditivo parece possuir um dispositivo que dispara um processo de reorganização perceptiva da cena, quando um evento súbito e significativo é detectado. Na experiência da música esse processo é motivado por mudanças relevantes em sua compo- 
sição tonal, por redefinição de simetrias e assimetrias temporais e posicionais, e por variações espectrais, que podem então ser entendidas como gatilhos para a reorganização perceptiva do fluxo musical. Como o meio ambiente e, em particular, o fluxo musical está em contínuo e permanente estado de mudança, devemos nos perguntar como distinguimos mudanças graduais e progressivas de mudanças súbitas. Se entendermos uma mudança súbita como um evento musical que supera abruptamente um limiar de discrepância em um ou mais dos domínios acústicos acima referidos - anulando instantaneamente qualquer possibilidade de experiência de progressão -, devemos nos perguntar então o que, propriamente, constitui uma mudança súbita e significativa no meio, uma novidade percebida como indicativa do surgimento de um novo evento e não uma transformação gradual de um evento já presente.

Na experiência comum da cena auditiva agrupamos em um único fluxo os eventos que percebemos ser emitidos por uma mesma fonte; empregamos esse mesmo recurso para agrupar sequencialmente os eventos musicais que nos parecem refletir regularidades tonais, texturais, temporais e posicionais. Uma sequência de eventos oriundos de uma mesma fonte geralmente apresenta uniformidade ou mudanças progressivas e coerentes ao longo do tempo. Uma sequência de notas em movimento melódico coerente é assim percebida, porque apesar de individuá-las pelas súbitas e consecutivas mudanças de conteúdo tonal, percebemos outros parâmetros, tais como os constituintes espectrais, significativamente regulares, o que, de outro modo, resultaria na percepção da presença de várias fontes. De acordo com os resultados de mais de duas décadas de pesquisas em torno da percepção do fluxo auditivo, um evento auditivo é sempre e de alguma forma percebido como componente de um fluxo; e só pode ser percebido, em dado instante, como pertencente a um e apenas um fluxo - embora em alguns casos de "elisão" em música, possamos discutir a possibilidade de percepção dupla.

O que pretendo aqui colocar em discussão não é um modelo conceitual que nos permita prever antecipadamente - como regras - "o que” será identificado pelas respostas de orientação musicais como eventos mais significativos em dado fluxo musical. O modelo que o presente estudo pretende desenvolver tem como objeto revelar quais eventos e explicar por que tais eventos do fluxo musical se destacaram como pistas acústicas - a partir das quais o sistema perceptivo desenvolve processos de agrupação complexos e, sobretudo, processos de segregação - que resultaram no entendimento musical declarado por dado ouvinte. Se o modelo poderá ou não oferecer recursos expressivos eficazes para a elaboração composicional ou interpretativa será objeto de outra investigação.

\section{Considerações finais}

A título de exemplificação, examinarei sucintamente alguns dos fatores que regulam as respostas de orientação diante de descontinuidades provocadas por transientes sensoriais no fluxo de uma pequena peça de Luciano Berio para contrabaixo solo, intitulada Psy (1989). Considerarei (1) os processos atencionais baseados em "objeto" e em "posição espacial”, (2) a classe de parâmetros bottom-up que influenciam o controle atencional, (3) o princípio da conectividade uniforme que rege o particionamento do fluxo musical por "contornos" e "regiões", e (4) os transientes sensoriais do fluxo musical relacionados a mudanças de conteúdo tonal, textural, temporal e topográfico dos componentes do fluxo.

Os fatores tonais parecem estar no centro da elaboração formal de Berio neste trabalho, pois o entendimento da peça está intimamente relacionado a tensões e contrastes 
oriundos de sua harmonicidade. A seção inicial (c.1-24) apresenta forte coerência tonal, resultante do emprego sistemático do conjunto $\mathrm{T}_{7}(02357)$ - pentacorde menor - em uma única configuração (voicing). Há cinco movimentos melódicos ascendentes de $\mathrm{Sol}_{2}$ a Rée ${ }_{3}$, imediatamente seguidos de movimentos compensatórios em direção contrária (de mesmo âmbito). As durações dos movimentos - sinalizadas na Figura 1 em número de semicolcheias - é progressivamente menor até sua última reprodução, quando reassume duração similar à inicial. Cumpre observar que as sucessivas asserções do movimento ascendente contribuem decisivamente para a habituação do conteúdo tonal do fluxo; somente no compasso final da seção (c.24) é que o autor quebra a homogeneidade tonal estabelecida, inserindo um simples "transiente" (a nota Lá 2 bemol) que implica descontinuidade tonal e potencial resposta de orientação do ouvinte no domínio tonal.

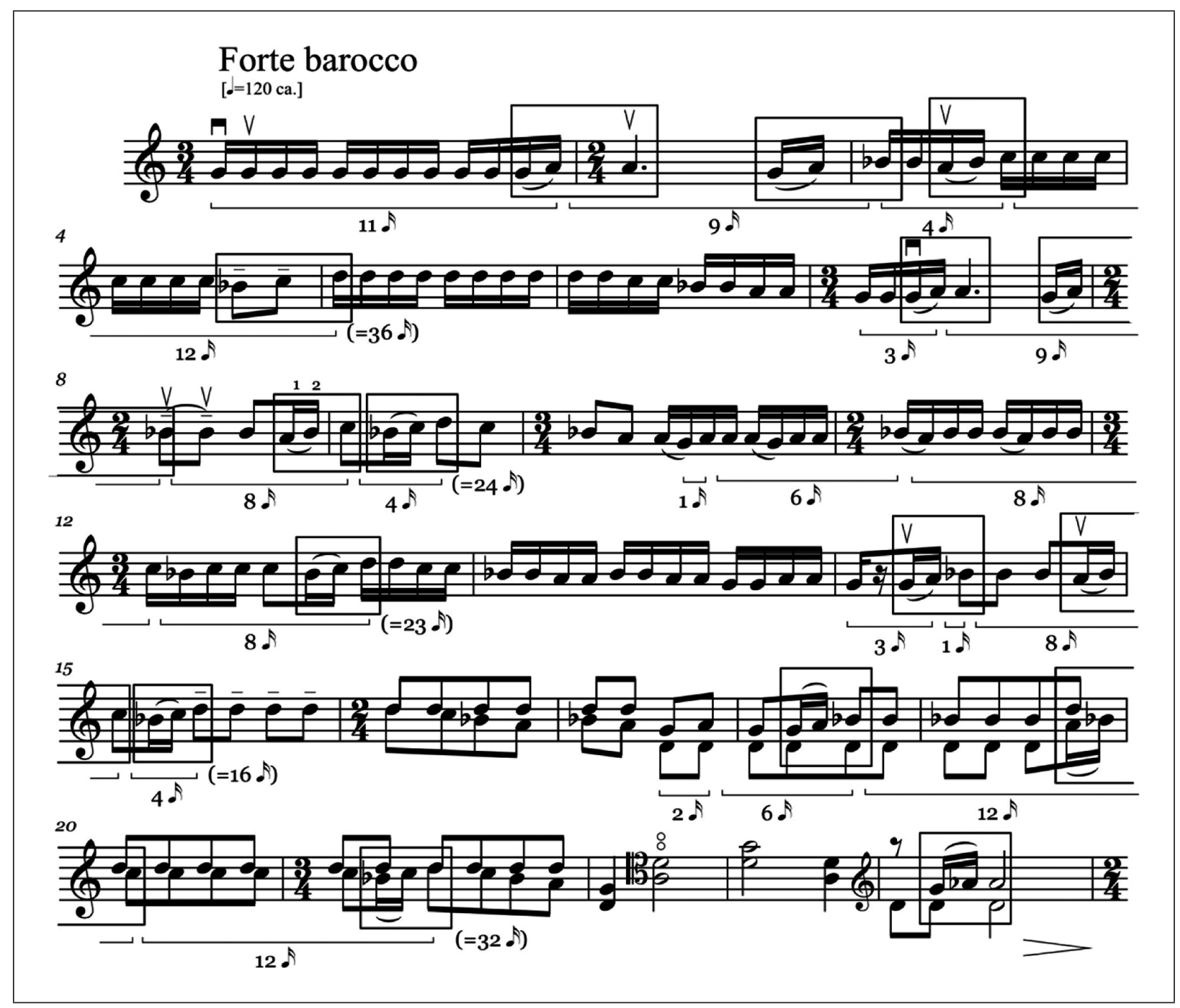

Figura 1: Trecho inicial de Psy, para contrabaixo solo, de Luciano Berio.

É importante atentar que a experiência de movimento - no caso em questão, movimento melódico - é uma propriedade emergente da variação conjunta de "conteúdo tonal" dos eventos simples agrupados sequencialmente (notas) e "duração" desses eventos sequenciais (tempo de permanência na escuta). No fluxo contínuo da música percebemos movimentos todo o tempo, segregados entre si ou não. Contudo, movimentos mais "simples" e 
"regulares" destacam-se por pregnância, constituindo-se referências formais importantes na construção do sentido musical. O trecho exibido pela Figura 1 desenrola-se com ênfase em amplos movimentos tonais ascendentes - que fazem precipitar tensões posicionais entre seus extremos -, mas uma "figura" em particular se destaca por pregnância e podemos denominá-la motivo principal (sinalizado por caixas na Figura 1). O que aqui denomino "figura" é, pois, um movimento pregnante, um evento pontual de agrupação cuja repetição conforma uma simetria de padrão, tornando-se potencialmente relevante como pista acústica para respostas de orientação - e cuja consequente habituação constitui um dos principais elementos de coerência do fluxo musical. Ainda enfocando o aspecto motor do fluxo, a predominância de constância e intensidade de atividade articulatória, durante esta seção inicial da peça, também faz ressaltar como pistas acústicas relevantes para respostas de orientação as interrupções de continuidade percebidas nas duas primeiras apresentações do movimento pentacordal (compassos 2 e 7). A partir da segunda interrupção, o movimento mantém-se constante até os eventos de fechamento da seção (c.22-24) - desmobilização por redução drástica da atividade articulatória.

Outra classe de respostas de orientação é provocada por transientes relacionados a variações espectrais, que acompanham todo o arco melódico final, a partir do compasso 16 e até o final da seção representada na Figura 1. Trata-se da descontinuidade do fluxo provocada por mudanças súbitas e significativas de timbre e densidade. Não destaco aqui a possibilidade de percepção de um fluxo musical segregado pelo ouvinte em dois subfluxos - o que pode ou não ser de fato experimentado pelos ouvintes -, a partir do adensamento textural proporcionado pelo emprego de cordas duplas. Destaco o explícito aumento de densidade do fluxo musical, natural e instantaneamente percebido pelo ouvinte como descontinuidade, o que torna o evento pontual que estabelece este contraste um indicador relevante de orientação da atenção no processo de construção do sentido musical.

Na experiência da música o ouvinte tem sua atuação restringida pela transitoriedade dos eventos musicais que fluem em complexos sonoros em geral de notável potencial de estimulação. A hipótese que fundamenta o modelo conceitual em desenvolvimento no presente estudo é que as respostas de orientação musicais podem ser consideradas um gatilho para o entendimento da música no ato da escuta. Entendo que as operações cognitivas provocadas pela percepção de "eventos relevantes" no fluxo musical constituem a etapa inicial do processo de entendimento musical, que se completará com a confrontação dos mesmos com esquemas cognitivos memorizados, condicionando assim toda produção de sentido na experiência da música. O entendimento estaria intimamente associado a tensões, cuja percepção é regulada pelas affordances do texto musical e pelas competências do ouvinte, tendo em vista sua maior ou menor fluência perceptiva com os padrões apresentados pela obra - e que implicam expectativa e antecipação. Argumento aqui que no processo de conceituação do nosso entendimento de uma narrativa tensional na música percebida em performance (esta tanto como atividade de um performer quanto como produção imaginativa de um ouvinte) hierarquizamos os atributos daquilo que dizemos ser "a obra" dispostos em dimensões perceptivas interdependentes, relacionadas a fatores tonais, texturais, temporais e topográficos dos componentes da cena musical, identificados em nossas respostas de orientação.

Assim sendo, acredito que um modelo conceitual baseado na superposição de pistas linguísticas e pistas acústicas, ou seja, no cotejamento do entendimento proposicional de dado trecho musical com o mapeamento das possíveis respostas de orientação que condicionam o entendimento declarado pelo ouvinte, pode revelar perspectivas importantes do percurso semântico por ele realizado. Isto demanda o desenvolvimento de protocolo expe- 
rimental que envolva tanto processos de identificação das pistas acústicas percebidas como relevantes em dada experiência musical - reguladas por suas discrepâncias potenciais no fluxo, bem como pelas inferências esquemáticas de ouvintes situados e culturalmente determinados - quanto monitoramentos de alterações neurofisiológicas significativas durante a experiência, dados estes que podem corroborar os resultados daqueles processos.

\section{Notas}

1 Esquema é uma estrutura conceitual de alto nível que organiza nossas experiências prévias, e a partir da qual interpretamos as novas situações (MINSKY, 1975; RUMELHART, 1980). Assim entendidos, os esquemas seriam sínteses de abstração dos componentes mais estáveis e essenciais de nossas experiências. Com este dispositivo cognitivo estaríamos mais capacitados a processar rapidamente as "novas informações" que o meio circundante nos oferece.

2 Tradução livre de: Attention has been used to refer to all those aspects of human cognition that the subject can control and to all aspects of cognition having to do with limited resources or capacity, and methods of dealing with such constraints.

3 A descrição original de Gibson para affordance é surpreendentemente simples: As affordances do meio ambiente são aquilo que ele oferece ao animal, o que ele proporciona, "seja para o bem ou para o mal", a qualquer animal que seja capaz de percebê-lo e usá-lo; enfim, mediante suas propriedades (affordances) o meio oferece oportunidades para comportamentos particulares. Alguns preferem argumentar que as affordances não são propriedades do meio e nem sequer propriedades. Para Chemero (2003), affordances são relações entre aspectos particulares dos animais e aspectos particulares das situações.

\section{Referências}

ATCHLEY, P.; KRAMER, A. F.; HILLSTROM, A. P. Contingent capture for onsets and off-sets: Attentional set for perceptual transients. Journal of Experimental Psychology: Human Perception \& Performance, 26, 594-606, 2000.

BREGMAN, A. S. Auditory scene analysis: the perceptual organization of sound. Cambridge, MA: MIT Press, 1990.

BREGMAN, A. S. Using brief glimpses to decompose mixtures. In J. SUNDBERG; L. NORD; R. CARLESON (Eds.), Music, language, speech and brain (p. 284-293). London: Macmillan, 1991.

CANNY, J. F. A computational approach to edge detection. IEEE Transactions on Pattern Analysis and Machine Intelligence, 8, 769-798, 1986.

CHEMERO, A. An outline of a theory of affordances. Ecological Psychology, 15(2), 181-195, 2003.

DESIMONE, R.; DUNCAN, J. Neural mechanisms of selective visual attention. Annual Review of Neuroscience, 18, 193-222, 1995.

DOWNING, P. E. Interactions between visual working memory and selective attention. Psychological Science, v. 11, n. 6, 2000.

DUNCAN, J. Selective attention and the organization of visual information. Journal of Experimental Psychology: General, 113, 501-517, 1984.

DUNCAN, J.; HUMPHREYS, G. Visual search and stimulus similarity. Psychological Review, 96, 433-458, 1989.

EGLY, R.; DRIVER, J.; RAFAL, R. D. Shifting visual attention between objects and locations: Evidence from normal and parietal lesion subjects. Journal of Experimental Psychology: General, 123, 161-177, 1994. 
FOLK, C. L.; REMINGTON, R. Can new objects override attentional control settings? Perception \& Psychophysics, 61, 727-739, 1999.

GIBSON. J. J. The theory of affordances. In: SHAW, R.; BRANSFORD, J. (Eds.), Perceiving, acting, and knowing: Towards an ecological psychology. New Jersey: Lawrence Erlbaum Associates, p. 67-82, 1977.

GIBSON, James J. The ecological approach to visual perception. Houghton Mifflin, 1979.

GIBSON, B. S.; KELSEY, E. M. Stimulus- driven attentional capture is contingent on attentional set for display wide visual features. Journal of Experimental Psychology: Human Perception \& Performance, 24, 699-706, 1998.

GRASTYAN, E. The significance of the earliest manifestations of conditioning in the mechanism of learning. In A. FESSARD et al. (Eds.), Brain Mechanisms and Learning. Oxford: Blackwell, 1961.

HARTMANN, W. M. Pitch perception and the segregation and integration of auditory entities. In G. M. EDELMAN, W. E. GALL, \& W. M. COWAN (Eds.), Auditory function (p. 623-645). New York: Wiley, 1988.

HARTMANN, W. M.; MCADAMS, S.; SMITH, B. K. Hearing a mistuned harmonic in an otherwise periodic complex tone. Journal of the Acoustical Society of America, 88, 1712-1724, 1990.

HURON, D. Sweet anticipation: Music and the psychology of expectation. Cambridge, MA: MIT Press, 2006.

JONES, M. R.; YEE, W. Attending to auditory events: The role of temporal organization. In S. MCADAMS \& E. BIGAND (Eds.), Thinking in sound: The cognitive psychology of human audition (p. 69-112). Oxford: Oxford University Press, 1993.

JONIDES, J.; YANTIS, S. Uniqueness of abrupt visual onset in capturing attention. Perception \& Psychophysics, 43, 346-354, 1988.

KAHNEMAN, D.; HENIK, A. Effects of visual grouping on immediate recall and selective attention. In S. DORNIC (Ed.), Attention and performance: Vol. 2, Proceeding of the Doders centenary symposium on reaction time (p. 307-331). NJ: Erlbaum, 1977.

KOFFKA, K. Principles of Gestalt Psychology, New York, Harcourt, Brace \& World, 1935/1963.

KÖHLER, W. Gestalt Psychology, New York, New American Library, 1947/1980.

KRAMER, A. F.; JACOBSEN, A. Perceptual organization and focused attention: The role of objects and proximity in visual processing. Perception \& Psychophysics, 50, 267-284, 1991.

LEUNG, T.; MALIK, J. Contour continuity in region-based image segmentation. In Proceedings of the 5th European Conference on Computer Vision. Freiburg, Germany, 1998.

LUCK, S.; VECERA, S. Attention. In H. PASHLER. Stevens' handbook of experimental psychology, $3^{\text {rd }}$ Ed., (p. 235-286). New York: John Wiley \& Sons, Inc., 2002.

MARR, D.; HILDRETH, E. C. Theory of edge detection. In Proceedings of the Royal Society of London: Series B, 207, 187-217, 1980.

MCADAMS, S. Segregation of concurrent sounds: I. Effects of frequency modulation coherence. Journal of the Acoustical Society of America, 86, 2148-2159, 1989.

MCADAMS, S. Recognition of sound sources and events. In S. MCADAMS \& E. BIGAND (Eds.), Thinking in sound: The cognitive psychology of human audition (p. 146-198). Oxford: Oxford University Press, 1993. 
MCADAMS, S.; BOTTE, M.-C.; DRAKE, C. Auditory continuity and loudness computation. Journal of the Acoustical Society of America, 103, 1580-1591, 1998.

MCADAMS, S.; BREGMAN, A. S. Hearing musical streams. Computer Music Journal, 3(4), 26-43, 1979.

MCADAMS, S.; DRAKE, C. Auditory perception and cognition. In p. 397-492, 2002.

MEYER, L. B. Emotion and meaning in music. Chicago: University of Chicago Press, 1956.

MINSKY, M. A framework for representing knowledge. In P. Winston (Ed.), The Psychology of Computer Vision. McGraw-Hill, 1975.

MOORE, B.; GLASBERG, B.; PETERS, R. Relative dominance of individual partials in determining the pitch of complex tones. Journal of the Acoustical Society of America, 77, 1853-1860, 1985.

PALMER, C.; KRUMHANSL, C. L. Pitch and temporal contributions to musical phrase perception: Effects of harmony, performance timing, and familiarity. Perception \& Psychophyics, 41, 505-518, 1987.

PALMER, S. E. Perceptual organization in vision. In H. PASHLER. Stevens' handbook of experimental psychology, $3^{\text {rd }}$ Ed., (p. 177-234). New York: John Wiley \& Sons, Inc., 2002.

PALMER, S.; ROCK, I. Rethinking perceptual organization: The role of uniform connectedness. Psychonomic Bulletin \& Review, 1(1), 29-55, 1994.

PAVLOV, I. Conditioned reflexes: An investigation of the physiological activity of the cerebral cortex. (Translated and edited by G. V. Anrep). London: Oxford University Press, 1927.

REISENZEIN, R., MEYER, W.-U., \& SCHÜTZWOHL, A. Reactions to surprising events: A paradigm for emotion research. In N. FRIJDA (Ed.), Proceedings of the $9^{\text {th }}$ conference of the International Society for Research on Emotions (p. 292-296). Toronto: ISRE, 1996.

REISENZEIN, R., MEYER, W.-U., \& NIEPEL, M. Surprise. In V. S. RAMACHANDRAN (Ed.), The Encyclopedia of Human Behavior, $2^{\text {nd }}$ Ed., v. 3, p. 564-570 (Amsterdam: Elsevier), 2012.

ROBERTS, B.; BREGMAN, A. S. Effects of the pattern of spectral spacing on the perceptual fusion of harmonics. Journal of the Acoustical Society of America, 90, 3050-3060, 1991.

ROSCH, E. Principles of categorization. In: E. ROSCH; B. LLOYD (Eds.) Cognition and categorization, 27-48. Hillsdale, N. J.: Erlbaum Associates, 1978.

ROSCH, E.; MERVIS, C. B.; GRAY, W.; JOHNSON, D.; BOYES-BRAEM, P. Basic objects in natural categories. Cognitive Psychology, 8, 382-439, 1976.

RUMELHART, D. E. Schemata: the building blocks of cognition. In: R. J. Spiro et al. (Eds.), Theoretical issues in reading comprehension. Hillsdale, NJ: Lawrence Erlbaum, 1980.

SECHENOV, I. Reflexes of the brain. Cambridge, Mass.: The MIT Press, 1863/1965.

SOKOLOV, E. N. Neuronal models and the orienting reflex. In Mary A. B. BRAZIER (ed.), The Central Nervous System and Behavior, p. 187-276. New York: Josiah Macy, Jr. Foundation, 1960.

SHIFFRIN, R. Attention. In R. ATKINSON, R. HERRNSTEIN, G. LINDZEY \& R. LUCE (eds.), Steven's handbook of experimental psychology. New York: John Wiley, p. 738-811, 1988.

SOKOLOV, E. N. Perception and the conditioned reflex. Oxford: Pergamon Press, 1963. 
HILLSTROM, A. P.; YANTIS, S. Visual motion and attentional capture. Perception \& Psychophysics, 55, 399-411, 1994.

VECERA, S. P.; FARAH, M. J. Does visual attention select objects or locations? Journal of Experimental Psychology: General, 123, 146-160, 1994.

VECERA, S. P. Toward a biased competition account of object-based segregation and attention. Brain and Mind, 1, 353-384, 2000.

WERTHEIMER, M. Gestalt Theory. In Willis D. ELLIS (Ed.) (1938/1997). A Source Book of Gestalt Psychology. Highland, NY: Gestalt Journal Press, 1924.

YANTIS, S. Goal-directed and stimulus - driven determinants of attentional control. In S. MONSELL \& J. DRIVER (Eds.), Attention and performance: Vol. 18, Control of cognitive processes (p. 73-103). Cambridge: MIT Press, 2000.

YANTIS, S.; EGETH, H. E. On the distinction between visual salience and stimulus-driven attentional capture. Journal of Experimental Psychology: Human Perception \& Performance, 25, 661-676, 1999.

ZERA, J.; GREEN, D. M. Detecting temporal onset and offset asynchrony in multicomponent complexes. Journal of the Acoustical Society of America, 93, 1038-1052, 1993. 